\title{
Virtual reality exposure-based therapy for the treatment of post-traumatic stress disorder: a review of its efficacy, the adequacy of the treatment protocol, and its acceptability
}

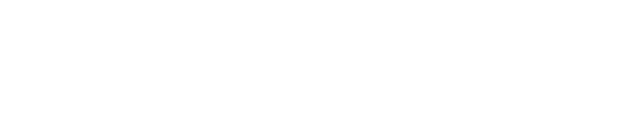

\author{
Cristina Botella' \\ Berenice Serrano' \\ Rosa M Baños ${ }^{2}$ \\ Azucena Garcia-Palacios' \\ 'Universitat Jaume I, Castellón de la \\ Plana, Spain; ${ }^{2}$ Universitat de Valencia, \\ Valencia, Spain
}

\begin{abstract}
Introduction: The essential feature of post-traumatic stress disorder (PTSD) is the development of characteristic symptoms following exposure to one or more traumatic events. According to evidence-based intervention guidelines and empirical evidence, one of the most extensively researched and validated treatments for PTSD is prolonged exposure to traumatic events; however, exposure therapy can present some limitations. Virtual reality (VR) can help to improve prolonged exposure because it creates fictitious, safe, and controllable situations that can enhance emotional engagement and acceptance.
\end{abstract}

Objective: In addition to carrying out a review to evaluate the efficacy of VR exposure-based therapy (VR-EBT) for the treatment of PTSD, the aim of this study was to contribute to analyzing the use of VR-EBT by: first, evaluating the adequacy of psychological treatment protocols that use VR-EBT to treat PTSD; and second, analyzing the acceptability of VR-EBT.

Method: We performed a replica search with descriptors and databases used in two previous reviews and updated to April 2015. Next, we carried out an evaluation of the efficacy, adequacy, and acceptability of VR-EBT protocols.

Results: Results showed that VR-EBT was effective in the treatment of PTSD. The findings related to adequacy showed that not all studies using VR-EBT reported having followed the clinical guidelines for evidence-based interventions in the treatment of PTSD. Regarding acceptability, few studies evaluated this subject. However, the findings are very promising, and patients reported high acceptability and satisfaction with the inclusion of VR in the treatment of PTSD.

Conclusion: The main weaknesses identified in this review focus on the need for more controlled studies, the need to standardize treatment protocols using VR-EBT, and the need to include assessments of acceptability and related variables. Finally, this paper highlights some directions and future perspectives for using VR-EBT in PTSD treatment.

Keywords: evidence-based intervention, prolonged exposure, treatment efficacy

\section{Introduction}

According to the DSM-5, ${ }^{1}$ post-traumatic stress disorder (PTSD) involves the development of characteristic symptoms, such as distressing memories or dreams about the traumatic event, flashbacks, psychological distress produced by internal or external cues that symbolize the traumatic event, physiological reactions, avoidance of associated stimuli, and negative alterations in cognitions and mood. These symptoms appear after exposure to one or more traumatic events (eg, exposure to war as a combatant or civilian, a physical attack, robbery, mugging, childhood abuse, sexual violence,
Correspondence: Cristina Botella Av Vicente Sos s/n, Edif de Investigación II, Universitat Jaume I, Castellón de la Plana 12006, Spain Tel +34964387639

Email botella@uji.es 
being kidnapped, a terrorist attack, natural or human-made disasters, and motor vehicle accidents). Prevalence in the United States is around $8.7 \%$. Rates of PTSD are higher among veterans and other people whose vocation increases the risk of traumatic exposure (eg, firefighters) and among rape survivors. PTSD is more prevalent in females. Several studies have found an association between PTSD and low quality of life. ${ }^{2}$

One of the most commonly used evidence-based interventions for PTSD is prolonged exposure, ${ }^{3-5}$ which is based on emotional processing theory. ${ }^{6}$ The main components of prolonged exposure are in vivo and imaginal exposure to stimuli related to the traumatic experience, in addition to psycho-education and controlled breathing. In vivo exposure consists of gradually and systematically approaching situations that patients avoid. Imaginal exposure involves revisiting the memory in the imagination and recounting the traumatic event. ${ }^{5,7}$ Prolonged exposure's efficacy has been demonstrated in several studies, ${ }^{8-15}$ and has been compared to other therapeutic techniques, such as stress inoculation training or supportive counseling; ${ }^{16}$ stress inoculation training or combined treatment; ${ }^{17}$ and cognitive restructuring. ${ }^{18}$ A recent review concludes that, in general, the addition of these techniques does not enhance the efficacy of prolonged exposure. This review also points out that, despite all these findings, many people with PTSD receive treatments with unknown efficacy. ${ }^{5}$

Prolonged exposure is an efficacious treatment. However, some people have difficulty imagining the traumatic event or emotionally engaging with the memory of the traumatic event. ${ }^{5}$ In addition, some people may find it aversive, given that it involves confronting feared and avoided memories. ${ }^{19}$

Another issue is that exposure therapy appears to be under-utilized in clinical practice. Becker et $\mathrm{al}^{20}$ found that, although about half of a sample of clinicians reported being familiar with imaginal exposure, only 17\% used it to treat PTSD. One of the main reasons for not using exposure was a lack of training and experience with PTSD. When analyzing trained clinicians with experience in treating PTSD, less than $50 \%$ used exposure to treat their PTSD patients. The barriers reported were concerns about worsening the symptoms or increasing the dropout rate. However, these concerns are not supported by the scientific evidence. ${ }^{21}$ In summary, exposure therapy seems to have an important dissemination problem among clinicians.

Division 12 of the American Psychological Association considers prolonged exposure to be a psychological treatment with strong empirical support, ${ }^{22,23}$ which means it is supported by two well-designed studies conducted by independent investigators. ${ }^{3}$ Furthermore, according to Division 12 of the American Psychological Association, other therapies have also shown empirical support, such as present-centered therapy, cognitive processing therapy, seeking safety (for PTSD with comorbid substance use disorder), and eye movement desensitization and reprocessing. Nevertheless, few studies have shown the efficacy of these other therapies. ${ }^{24}$

Several studies suggest that prolonged exposure has been studied more broadly and extensively than other therapies, ${ }^{11,12,17,18,24-28}$ positioning it as the treatment of choice. The National Institute for Clinical Excellence also recommends the use of prolonged exposure as the treatment of choice because it is considered the best-evaluated PTSD treatment focusing on exposure. ${ }^{4}$ Therefore, it is important to work on improving the dissemination of prolonged exposure and facilitate its use in people who have difficulties imagining the traumatic memory or engaging emotionally, in addition to increasing the acceptance of exposure therapy by both patients and clinicians. In this regard, technology, and specifically virtual reality (VR), can help to advance toward this goal.

\section{VR in the treatment of mental disorders}

VR is a technology that allows the simulation of different real-life situations in a tridimensional computer-generated environment in which the user can interact with the environment as if he/she were in the real world. VR has potential as an exposure technique for treating different mental disorders because VR and real objects have similar characteristics, which creates the illusion that the user is immersed and engaged with objects in the real world. From a psychological point of view, this is a powerful therapeutic tool that helps people change their behaviors and protects them while the change occurs. VR can help to modify behaviors, thoughts, and emotions through virtual experiences designed for and adapted to the person's needs, in order to facilitate and enhance the process of change. ${ }^{29}$ Various studies have demonstrated the efficacy of VR exposure-based therapy (VREBT) for treating different mental disorders. For example, a meta-analysis ${ }^{30}$ compared controlled studies using VR-EBT as a stand-alone treatment vs in vivo exposure therapy and a control group, for the treatment of anxiety disorders. Results showed that in vivo exposure therapy and VR-EBT were equally effective. Another meta-analysis ${ }^{31}$ also supports the efficacy of VR-EBT. The authors found that posttreatment 
results showed similar efficacy to cognitive behavioral therapy. VR-EBT shows a powerful real-life impact and good stability of the results over time, similar to that of evidencebased interventions. Other meta-analyses also support VREBT's efficacy in anxiety disorders. ${ }^{32,33}$

The main conclusions extracted from the literature (on the use of VR-EBT in the treatment of psychological disorders ${ }^{34-37}$ ) report that its main advantage is the therapist's degree of control over the situations, as he/she can prevent the occurrence of unpredictable events or repeat a specific exposure task as many times as necessary. Other benefits are the ability to expose the patient to stimuli and places that would otherwise be difficult to access (eg, a plane), or its usefulness in maximizing the benefits of in vivo exposure therapy. VR-EBT can be an alternative for people who find in vivo exposure therapy aversive. VR-EBT can offer a more confidential setting than in vivo exposure therapy (the patient's exposure task takes place within the discreet confines of the therapist's office). VR is a flexible tool because it can replicate different physical or situational environments to treat various mental disorders.

In fact, all of these advantages can facilitate the acceptability of exposure therapy. A study focused on this issue ${ }^{38}$ found that $76 \%$ of patients reported being more willing to be involved in VR-EBT. In another study conducted to find out therapists' perceptions of the benefits and costs related to the use of VR, the therapists reported having knowledge about VR and being interested in using it. ${ }^{39}$

The literature also addresses the limitations of the use of VR-EBT in mental health. ${ }^{34-37}$ Some of the most important problems include therapists' limited accessibility to systems based on VR-EBT and lack of training in the use of this technology. In addition, there is a need for more cross-cultural studies to facilitate the generalization of the VR-EBT effects, and studies with long-term follow-ups would help to assess the maintenance of the results.

\section{VR-EBT in the treatment of PTSD}

In the specific case of PTSD, VR-EBT is a promising adjunct tool that is an alternative to prolonged exposure (in vivo and imagination) and is supported by various studies analyzed in recent reviews. ${ }^{40-43}$ VR can create fictitious, safe, and controllable situations that may be more acceptable to participants than in vivo exposure. In addition, VR can be an adequate alternative for cases where patients have limited ability to imagine $^{44}$ because VR provides the patient with visual and auditory stimuli, eliminating the need for intense imaginal skills and facilitating immersion in the traumatic event.
Currently, some VR systems already exist for the treatment of PTSD. Since the publication of the first case study using VR-EBT to treat this problem in $1999,{ }^{45}$ the number of studies has increased considerably. In the literature, two types of VR environments for the treatment of PTSD are clearly identified: 1) a VR environment with very specific and realistic situations (eg, Rothbaum et al and other studies ${ }^{45-52}$ ); and 2) a flexible VR environment that uses symbolism to represent any traumatic event. ${ }^{53}$ The specific and realistic VR environments were developed to treat specific traumatic events, such as the wars in Vietnam; ${ }^{45} \mathrm{Iraq}^{54}$ or Baghdad; ${ }^{51}$ or the terrorist attacks of September $11 .{ }^{48}$ Their main strength is their hyperrealism because they recreate the traumatic situation with very specific details. Their main weakness is that the purpose of these VR environments was to treat the traumatic events for which they were developed. As stressors that cause traumatic events can differ (eg, wars, sexual assault, terrorist attacks, natural disasters, etc), each traumatic event would require a specific VR environment. In terms of technological development costs, this would involve a major economic investment. ${ }^{55}$ The other type of VR environment is a flexible and adaptable system where any traumatic event can be "symbolically" represented using different tools (eg, symbols, pictures, music, sounds, video, etc). This VR environment could be a good solution for the limitations of VR environments that can only be used to treat a specific traumatic event. ${ }^{53}$ This VR environment is the first one reported in the literature with these characteristics. Its main strength is its flexibility in representing any traumatic event. Its main weakness is that for some PTSD populations, a more specific and realistic VR environment might be more suitable.

Recently, two reviews examined the efficacy ${ }^{41}$ and methodological quality ${ }^{40}$ of studies using VR-EBT to treat PTSD. The main conclusion of these studies is that VR-EBT is as efficacious as traditional exposure therapy. However, there is a need for more research that includes well-specified randomization procedures, treatment adherence, assessor blinding, and intent-to-treat analyses.

The present paper will perform a review to evaluate the efficacy of VR-EBT for the treatment of PTSD, but it will also address two additional issues in order to contribute to the analysis of the use of VR-EBT. First, we will evaluate the adequacy of the treatment protocols that use VR-EBT for PTSD, and then we will analyze the acceptability of VR-EBT.

\section{Method}

This paper presents a review based on the main findings about using VR-EBT in PTSD treatment, including studies that use 
VR-EBT and compare it to another treatment or a wait-list control. Then, we evaluate the adequacy and acceptability of the VR-EBT protocols.

\section{Procedure}

First, we performed a replica search with the same descriptors and databases used in two previous reviews, ${ }^{40,41}$ updated to April 2015 in the CINAHL, Google Scholar, ISI Web of Knowledge, Medline, PsycNET, and Scopus databases. Keywords or subjects related to the problem ("post-traumatic stress disorder", "stress disorder", "PTSD”, "combat experience", "emotional trauma", "stress reactions", and "trauma"), the intervention ("virtual reality", "computer simulation", "computer applications", and "human machine systems"), and comparison and outcomes ("treatment", "therapy", "intervention", "outcome", "comparison", and "control group") were used.

\section{Exclusion criteria}

The exclusion criteria were: 1) studies published in a nonpeer-reviewed journal, published as conference proceedings, or published as a book chapter; 2) studies that evaluated the same protocol and/or variables as a previous study, but without providing additional data; 3) VR-EBT was not compared to another treatment or a wait-list control; 4) VR was not used for treatment or was not specified (eg, assessment, usability studies, or 3D systems that were not designed specifically for VR-EBT); 5) treatments conducted on web-based systems; and 6) theoretical reviews or meta-analyses.

Specifically, the criterion of being published in a peerreviewed journal was selected because of the advantages cited in the literature: ${ }^{56}$ it plays a vital role in publishing because it allows control over scientific information; it improves the quality of a published paper, making it possible to select the best papers from journals, facilitating the detection of plagiarism. The cited study ${ }^{56}$ measured the attitudes of researchers toward peer review, finding that the peer review process is highly regarded by the majority of researchers and considered by most to be essential to the communication of research.

\section{Inclusion criteria}

The inclusion criteria were: 1) studies applying VR-EBT and comparing it to another treatment or a wait-list control; and 2) studies that do not meet any of the exclusion criteria.

With the selected studies, a VR-EBT protocol evaluation was carried out based on the evidence-based interventions clinical guidelines ${ }^{3,457}$ and evidence-based interventions studies $^{5,16}$ to treat PTSD.

\section{Results}

An initial search showed 439 results that responded to the initial search criteria. In a first screening, 315 publications were excluded because they were published in a non-peerreviewed journal, published as conference proceedings, or published as a book chapter; or they were duplicates, abstracts, conference proceedings, or published in another different source. The first screening yielded 124 results, identified and distributed as follows: trials or controlled studies comparing VR-EBT to another treatment or wait-list control, $n=20$; case studies or case series, $n=16$; trials with only one sample using VR-EBT, $n=12$; VR was not used in the treatment, $n=38$; treatment conducted on a web-based system, $n=3$; and theoretical reviews or meta-analyses, $n=35$. A second screening excluded 112 papers due to not meeting the inclusion criteria or meeting some exclusion criteria (eg, studies where there was a previous study that evaluated the same protocol).

Finally, 12 studies met the inclusion criteria and were included in this review. In addition to the studies analyzed in two previous reviews, ${ }^{40,41}$ we identified four new efficacy studies. ${ }^{54,58-60}$ Figure 1 shows a summary of the study selection process.

\section{Efficacy of VR-EBT for PTSD Using VR-EBT to treat military personnel}

Most of the studies on the efficacy of VR-EBT were conducted with military personnel who were sent to war zones (eg, Vietnam, Iraq, Afghanistan). In an open trial, ${ }^{61}$ military personnel who were sent to the Vietnam war $(\mathrm{N}=9)$ were enrolled in a VR-EBT $(n=5)$ or a present-centered therapy group $(\mathrm{n}=4)$. Results showed significant mean improvement $(P<0.05)$ on the Clinician Administered PTSD Scale $(\mathrm{CAPS})^{62}$ at posttreatment and 6-month follow-up, but no significant interaction between time and treatment condition. In another study, ${ }^{59}$ participants with PTSD $(\mathrm{N}=19)$ were randomized to VR-EBT $(n=9)$ or imaginal exposure $(n=10)$ treatment and compared to a control group without PTSD $(\mathrm{n}=18)$; in addition, a functional magnetic resonance imaging was conducted. After treatment, VR-EBT reduced CAPS ${ }^{62}$ scores $(P<0.05)$, but the imaginal exposure group showed no significant changes. Nevertheless, findings showed significant change $(P<0.05)$ in both groups on the PTSD checklist score; in addition, both groups showed significant reductions in amygdala activation after treatment. There were no significant changes in the control group.

The efficacy of VR-EBT in active military stationed in Iraq and still exposed to a war zone was also studied in a 


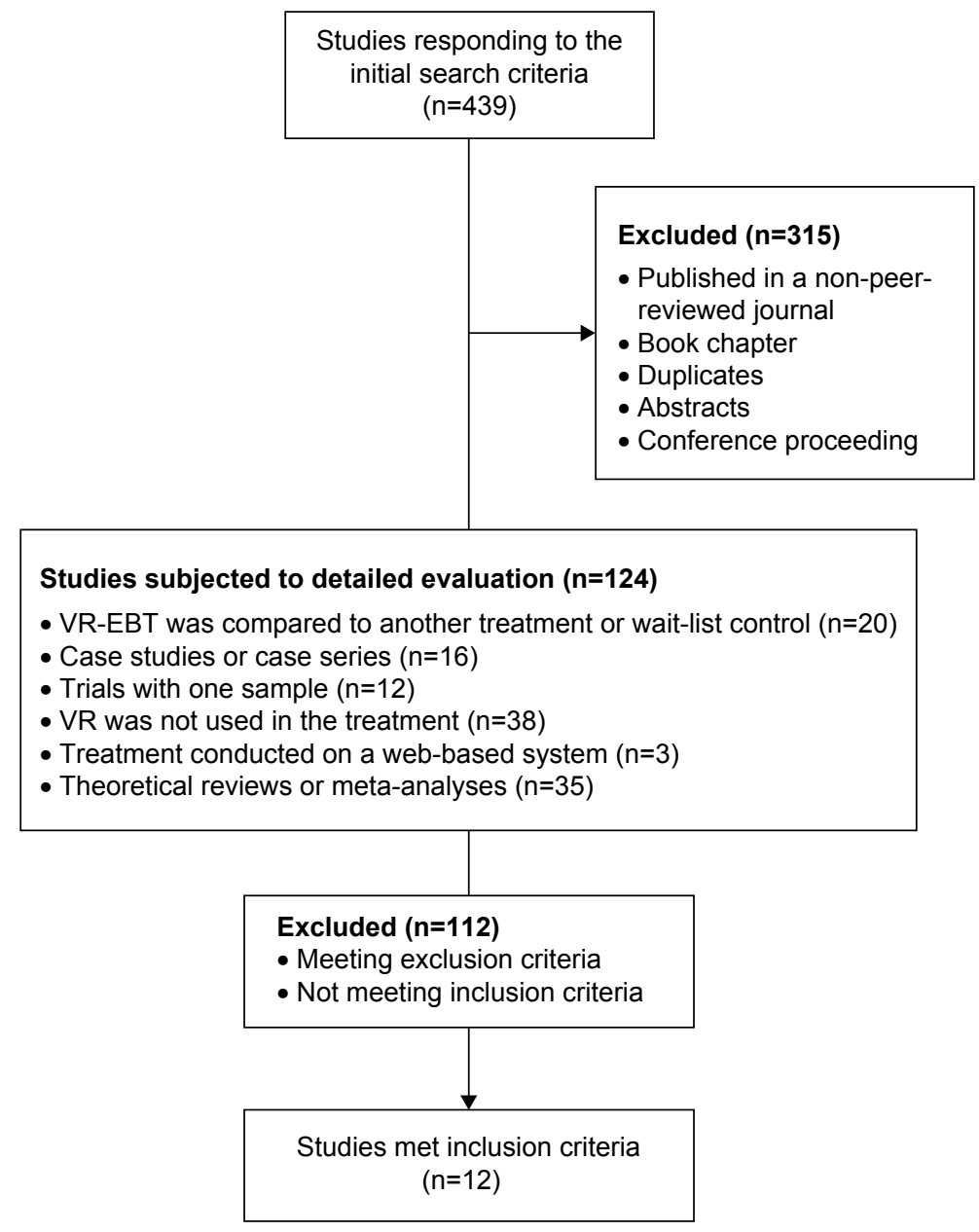

Figure I Summary of studies' selection process.

Abbreviations: EBT, exposure-based therapy; VR, virtual reality.

controlled nonrandomized trial $(\mathrm{N}=10)$ comparing VR-EBT $(n=6)$ to traditional exposure therapy $(n=4) .{ }^{63}$ All participants showed significant improvements in PTSD symptoms $(P<0.001)$, but without finding a significant interaction effect (VR-EBT vs traditional). A later randomized clinical trial $(\mathrm{N}=20)^{64}$ compared VR-EBT $(\mathrm{n}=10)$ to treatment as usual (prolonged exposure; cognitive processing therapy; eye movement desensitization and reprocessing; group therapy; psychiatric medication management; substance rehabilitation; inpatient services; or a combination of these) $(n=10)$. The results reported significant improvement $(P<0.01)$, but no significant differences between groups. A randomized controlled clinical trial $(\mathrm{N}=22)$ designed to treat combatrelated PTSD upon returning from the Iraq war ${ }^{65}$ compared the efficacy of VR-EBT $(n=12)$ to a wait-list control $(n=10)$. The results did not show significant differences on the CAPS $^{62}$ measure; but there was a significant decrease in criterion $\mathrm{C}$ in the VR-EBT group.
Another study developed a VR environment to treat military personnel stationed in African colonies. It validated this VR environment in a controlled trial $(\mathrm{N}=10)$ with three randomized groups: VR-EBT $(n=5)$, imaginal exposure $(n=2)$, and wait-list control $(n=3)$. Results showed no significant effects, but participants who received VR-EBT showed a reduction in symptoms associated with PTSD (depression and anxiety). ${ }^{50}$

\section{Using VR-EBT to treat victims from terrorist attacks}

Another population included in the field of VR-EBT consisted of victims of terrorist attacks. One study developed a VR environment as a therapeutic tool for victims of the September 11 terrorist attacks. This VR environment included different situations related to the attacks (eg, people jumping from the towers). A clinical trial $(\mathrm{N}=21)$ comparing VR-EBT $(n=13)$ to a wait-list control $(n=8)$ showed its efficacy. ${ }^{48}$ PTSD symptoms declined significantly at 
posttreatment $(P<0.01)$, maintaining these levels at the 6-month follow-up. The findings also revealed that significance between groups was also found $(P<0.01)$. This study included participants who showed no significant improvement using traditional imaginal exposure, which suggests that VR-EBT could be a useful alternative for patients who did not benefit from traditional exposure (in vivo or imaginal exposure). The authors suggest that the possibility of customizing and adjusting the exposure tasks in VR "may increase the patients' feelings of self-efficacy and of being an active agent in their own experience".

\section{Using VR-EBT to treat victims of criminal violence}

VR-EBT has also been administered to victims of criminal violence. A VR environment representing some unsafe places and situations (eg, assault) in Ciudad Juárez (Mexico) and Mexico City was developed and tested as a VR-EBT tool for treating people with PTSD caused by criminal violence. ${ }^{59}$ Participants $(\mathrm{N}=20)$ were randomized to the VR-EBT $(\mathrm{n}=10)$ or the prolonged exposure $(n=10)$ group. Results showed significant improvement on PTSD symptoms, anxiety, and depression in both groups $(P<0.05)$; there were no significant differences between groups.

\section{Using VR-EBT to treat victims of different traumatic events}

As mentioned above, there is another type of VR-EBT used in the treatment of PTSD. The main difference between this VR environment and the realistic VR environment is that it can be used to "symbolize" different traumatic events and does not require the use of a head-mounted display, making it very ergonomic and noninvasive for the patient. This VR environment is called "EMMA's World". This VR environment adapts the contents to the therapeutic needs of each patient and can be modified according to the emotional changes that occur during the therapeutic process. For a more detailed description, see Rey et al. ${ }^{66}$ The efficacy of this VR-EBT for treating PTSD was evaluated in a controlled trial ${ }^{55}$ with victims of different kinds of trauma (motor vehicle accidents, mobbing, robbery or assault, and domestic violence) $(\mathrm{N}=10)$. When comparing VR-EBT to cognitive behavioral therapy, symptoms declined significantly after treatment $(P<0.05)$. An analysis of the differential efficacy of the two treatments showed no significant differences. A later controlled trial ${ }^{67}$ confirmed the effectiveness of the VR-EBT in another sample $(\mathrm{N}=16)$ who had PTSD as a consequence of abuse, crime assault, and motor vehicle accidents. The results confirmed that VR-EBT ( $\mathrm{n}=8$ ) was as effective as cognitive behavioral therapy $(n=8)$. There were no significant differences between groups, but an important finding was that other associated symptoms (eg, depression or social interference) showed statistically significant differences in favor of VR-EBT.

\section{Using VR-EBT and pharmacological treatment}

Two recent studies explored the efficacy of combined treatments using VR-EBT and medication to enhance fear extinction. One of them analyzed efficacy of a treatment using VR-EBT and D-cycloserine in a randomized clinical trial. ${ }^{58}$ After their exposure to the September 11 terrorist attacks, participants $(\mathrm{N}=25)$ received $100 \mathrm{mg}$ of $\mathrm{D}$-cycloserine $(\mathrm{n}=13)$ or a placebo $(n=12) 90$ minutes before the VR-EBT sessions. PTSD remission rates were significantly greater for the VREBT and D-cycloserine group at posttreatment and at the 6-month follow-up. Another study ${ }^{54}$ compared the efficacy of a combined treatment using VR-EBT and D-cycloserine, alprazolam, or placebo. Veterans of the Iraq/Afghanistan war $(\mathrm{N}=156)$ were given VR-EBT plus $50 \mathrm{mg}$ of $\mathrm{D}$-cycloserine $(\mathrm{n}=53)$, VR-EBT plus $0.25 \mathrm{mg}$ of alprazolam $(\mathrm{n}=50)$, or VR-EBT plus placebo $(n=53)$. PTSD symptoms significantly improved across all conditions at posttreatment and at the 3-, 6-, and 12-month follow-ups. There were no differences in symptoms between D-cycloserine and placebo. Alprazolam and placebo differed significantly on the CAPS score at posttreatment and PTSD diagnosis at 3-month posttreatment; the alprazolam group showed a higher rate of PTSD than the placebo group.

\section{Adequacy of VR-EBT protocols}

Tables 1 and 2 summarize the identified findings, implementing five adequacy variables evaluated in each VR-EBT protocol, based on the evidence-based interventions clinical guidelines ${ }^{3,4,57}$ and evidence-based interventions studies (eg, Foa et $\mathrm{al}^{5,16}$ ) to treat PTSD. Table 3 shows the reported components of each treatment protocol by session.

\section{Acceptability of VR-EBT protocols}

The acceptability of the VR-EBT protocols for the treatment of PTSD is an important issue that has not been widely studied. Very few of the 48 studies reviewed that used VR-EBT to treat PTSD reported data about the acceptability of the VR-EBT protocols. In this section, we describe the studies that explored and reported acceptability.

A trial with a single sample ${ }^{68}$ assessed clients' satisfaction after a VR-EBT. The results indicated that all participants scored 30 or more on a scale ranging from 8 to 32, suggesting high levels of satisfaction with VR-EBT. 


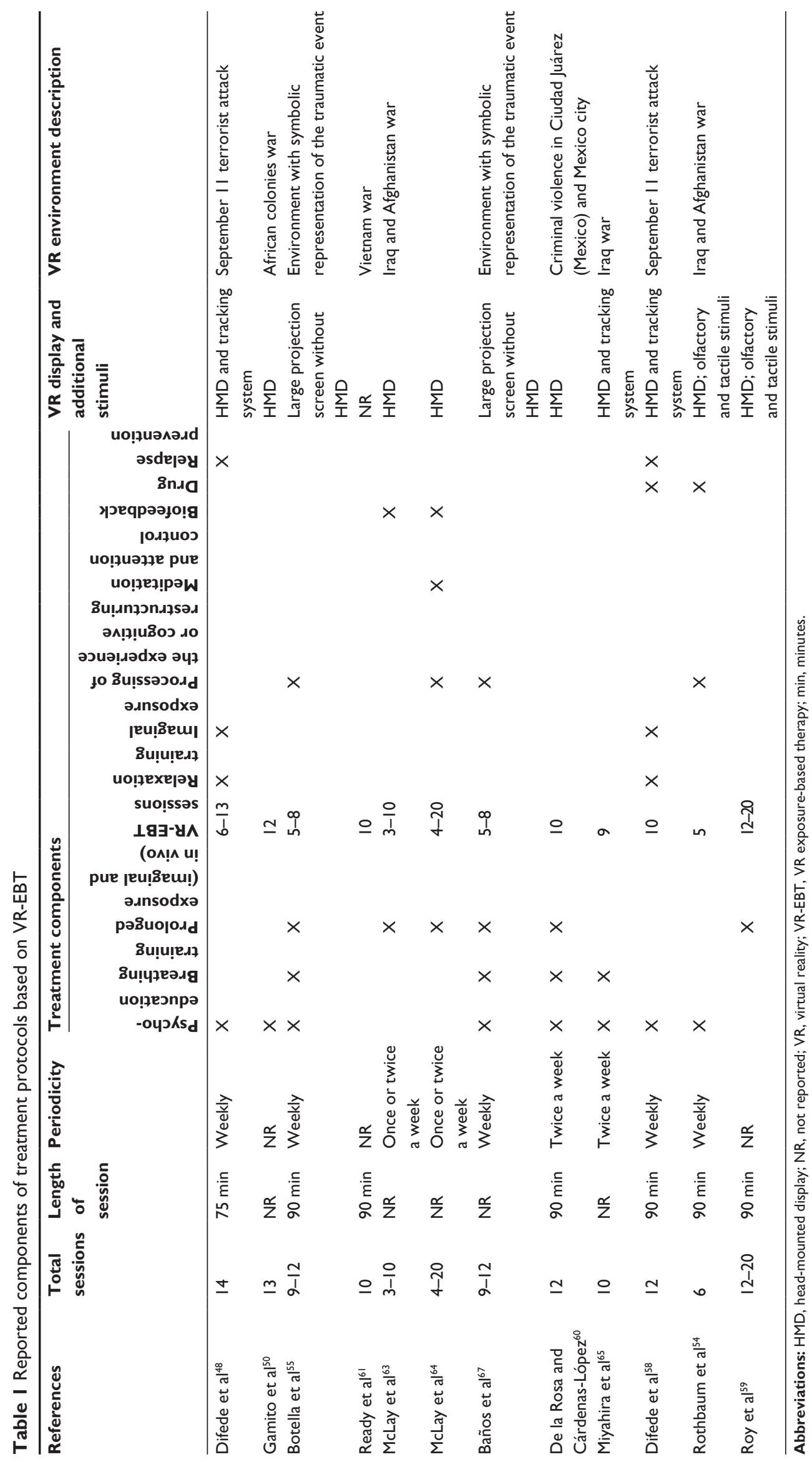


Table 2 Adequacy of VR-EBT protocols

\begin{tabular}{|c|c|c|}
\hline Adequacy variable & Description & Result \\
\hline I. Treatment of choice & $\begin{array}{l}\text { The use of prolonged exposure (with } \\
\text { in vivo and imaginal exposure), psycho- } \\
\text { education, and breathing training; it was } \\
\text { considered the treatment with a strong } \\
\text { research support, and studied more broadly } \\
\text { and extensively than other therapies. }\end{array}$ & $\begin{array}{l}\text { Six studies }{ }^{55,59,60,63,64,67} \text { reported the use of prolonged } \\
\text { exposure. }\end{array}$ \\
\hline 2. Number of sessions & A range from 8 to 12 is recommended. & $\begin{array}{l}\text { Total sessions ranged from } 3 \text { to } 20 \text {; six studies }{ }^{55,58,60,61,65,67} \\
\text { ranged from } 8 \text { to I } 2 \text { sessions; three studies }{ }^{59,63,64} \text { reported } \\
\text { that their protocol had a duration of } 3 \text { to } 20 \text { sessions. }\end{array}$ \\
\hline $\begin{array}{l}\text { 3. Treatment session } \\
\text { length }\end{array}$ & 90 minutes is recommended. & Six studies ${ }^{54,55,58-61}$ reported a length of 90 minutes. \\
\hline 4. Periodicity & Once a week is recommended. & $\begin{array}{l}\text { Seven studies }{ }^{48,54,55,58,63,64,67} \text { reported a weekly treatment } \\
\text { periodicity. }\end{array}$ \\
\hline $\begin{array}{l}\text { 5. (a) Number of sessions } \\
\text { of VR-EBT and (b) } \\
\text { the VR display and } \\
\text { additional stimuli used } \\
\text { were also addressed }\end{array}$ & It is not determined. & $\begin{array}{l}\text { (a) The VR-EBT range of sessions is between } 3 \text { and } 20 \text {. } \\
\text { (b) Nine studies } 48,50,54,58-60,63-65 \text { used a head-mounted display, } \\
\text { two studies }{ }^{55,67} \text { used a large projection screen, and one } \\
\text { study }^{61} \text { did not report this information. }\end{array}$ \\
\hline
\end{tabular}

Abbreviations: VR, virtual reality; VR-EBT, VR exposure-based therapy.

Baños et $\mathrm{al}^{53}$ also explored the acceptability of VR-EBT. They asked participants about their expectations and satisfaction with treatment. Before treatment, the mean scores ranged from 7 to 8 (on a $0-10$ scale) with regard to the logicality, satisfaction, or the utility of VR-EBT. In addition, participants rated aversiveness from 4 to 6 . After treatment, the satisfaction means increased compared to the expectation, as participants evaluated their satisfaction with VR-EBT program higher than they did initially, and they rated the treatment as less aversive.

Another study ${ }^{69}$ with veterans of the Iraq war evaluated their acceptance in using VR-EBT for mental health. A survey showed that $83 \%$ of the participants reported that they were neutral-to-very-willing to use some technology as part of a treatment; $71 \%$ were equally willing or more willing to use a treatment based on technology than to merely talk to a therapist in a traditional treatment.

De la Rosa and Cárdenas-Lópe ${ }^{60}$ explored differences in the level of satisfaction with the treatment between a group that received VR-EBT and another one that received imaginal exposure. The results showed no differences in the groups' satisfaction. This result coincides with another study ${ }^{70}$ that found no differences in satisfaction between the VR-EBT group and the imaginal exposure group. The participants evaluated both treatments as useful, and they would recommend them to a friend or family member who had PTSD. They also reported significant differences in the degree of aversion. The authors state that these findings are consistent with other studies using VR-EBT for specific phobias, where participants found it easier to take the first step to confront their fears in VR environments. ${ }^{38}$

Other studies that used VR-EBT for PTSD reported only qualitative or opinion data; for example, a case study ${ }^{71}$ reported that the participant said he felt comfortable with the technology and found the treatment to be logical and credible. In another case study, ${ }^{72}$ when the patient was asked his opinion of the role of VR in his improvement, he stated "I don't think I would be where I am today without it".

\section{Conclusion}

Currently, we have the possibility of applying VR-EBT for the treatment of PTSD. The literature review indicates that VR-EBT is an effective and promising alternative for the treatment of PTSD. Research reveals that VR-EBT is as efficacious as traditional treatments (imaginal exposure, in vivo exposure therapy, cognitive behavioral therapy, treatment as usual, and present-centered therapy). Furthermore, VR-EBT offers other advantages that could play an important role, for example, in reducing aversion to prolonged exposure, ${ }^{70}$ treatment dropout, and generalization of the results to other symptom groups, ${ }^{67}$ as well as long-term maintenance of the results. ${ }^{61,64,73}$ VR-EBT for the treatment of PTSD has shown a significant reduction in symptoms related to the traumatic event. Studies have focused mainly on war veterans, showing significant changes at posttreatment and follow-ups. The results from most of the studies are quite promising, showing that VR-EBT is useful for those suffering from acute or early 


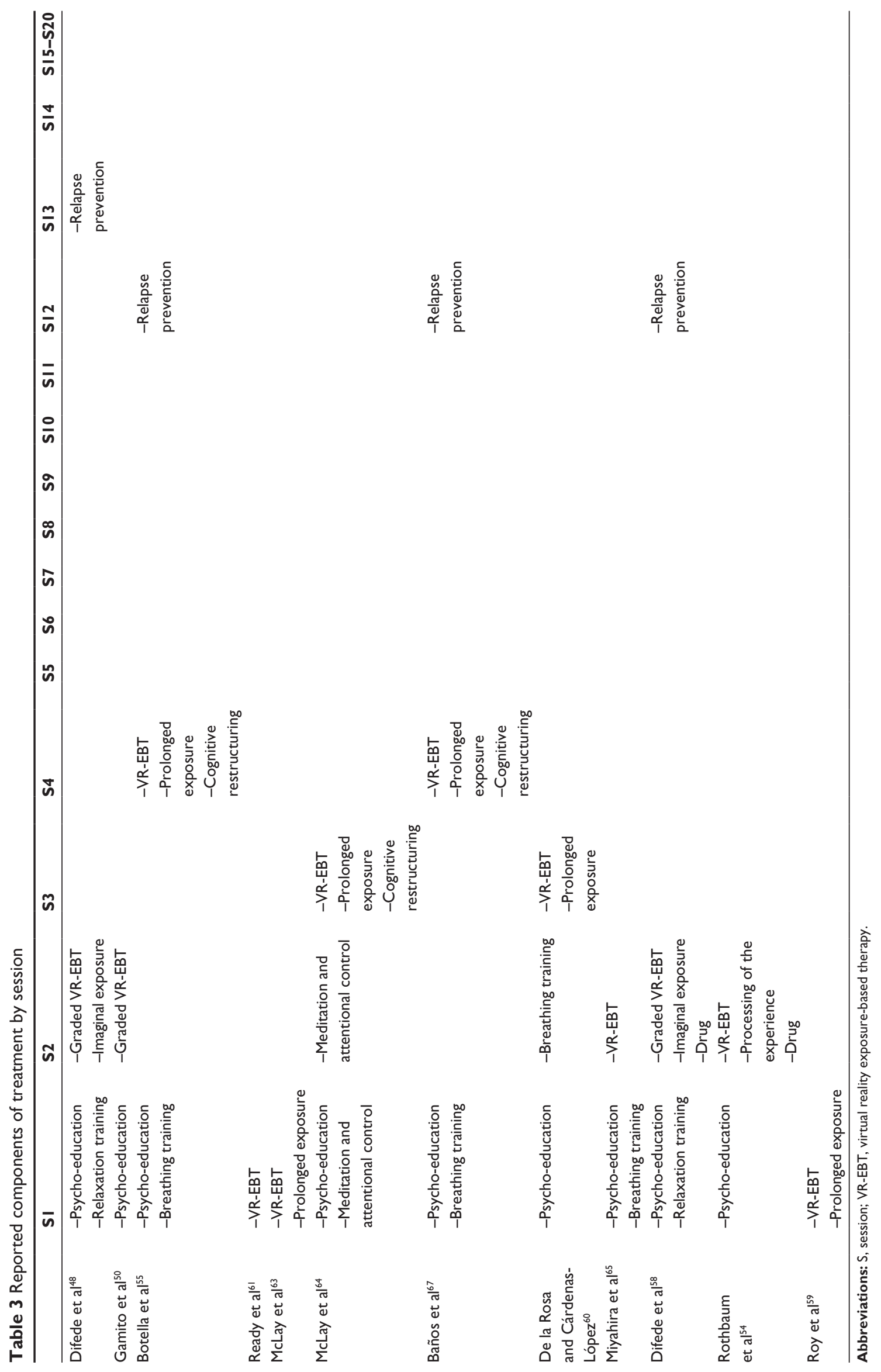


chronic combat PTSD. The findings also highlight that this treatment could be generalized to a wide range of symptoms and situations, with the greatest likelihood of long-term benefits. ${ }^{43}$ It is also important to note that controlled studies are still scarce. It is necessary to conduct more controlled trials with larger and more representative samples from different cultures in order to draw firmer conclusions about the effectiveness of VR-EBT in treating PTSD. We are likely to have additional data soon, given that a recent search found 13 trials registered on www.clinicaltrials.gov that specified the use of VR-EBT with or without other interventions (prolonged exposure, treatment as usual, drug, augmented exposure therapy, wait-list control) for treating PTSD; three of them were registered in the last 2 years.

Another relevant aspect is the use of VR from a prevention perspective. A study conducted with children in an earthquake-vulnerable area explored the possibilities of using VR to improve coping strategies to face future traumatic events. ${ }^{74}$ The results supported the hypothesis that pre-disaster VR programs can increase resilience in children. However, there is a lack of applications for VR-EBT aimed at the population of children. The literature only contains one reported case study ${ }^{75}$ about the treatment of PTSD in children, which reveals a great need in this field, considering the problems of abuse, violence, or natural disasters that children are exposed to around the world. Furthermore, recently, researchers are studying VR's potential in improving coping strategies and delivering stress resilience training in the military prior to traveling to war zones. ${ }^{76}$ Some authors highlight this potential use of $\mathrm{VR}^{43}$ as an emerging field of interest in mental health because it can prepare vulnerable populations for potential stressful and traumatic events.

The findings related to the adequacy of VR-EBT protocols showed that not all studies specified and reported that they followed the guidelines for evidence-based interventions in the treatment of PTSD. Most of them used VR-EBT alone or in combination with other therapeutic techniques, but there was no detailed report of all the features required by the clinical guidelines. ${ }^{3}$ Therefore, it is important to standardize treatment protocols for using VR-EBT in PTSD treatment, with an emphasis on treatment adherence.

Regarding its acceptability, one of the most frequently cited advantages of VR-EBT is that it would be more easily accepted than other forms of exposure (in vivo or imaginal). In fact, this has been explored and demonstrated by some studies in the field of anxiety disorders (eg, in specific phobia $^{38}$ ). However, and surprisingly, the field of PTSD treatment has not adequately addressed this question. One important finding of this review is that most of the studies did not include or report an assessment of acceptability. Few studies have evaluated this issue. The findings are very promising, as patients reported high satisfaction and acceptability about the inclusion of VR in PTSD treatment. Given that one of the limitations of exposure therapy for PTSD may be a lack of acceptance by patients and clinicians; ${ }^{20}$ it is important for studies to include an analysis of acceptability when exploring the utility of VR-EBT.

Another challenge is to provide training for mental health providers in the use of VR-EBT. This involves incorporating specific training in master programs. To date, very few universities or academic centers offer any specific training in the field of cyberpsychology or e-health. One study analyzed mental health clinicians' perceptions of using VR-EBT to conduct PTSD treatment in veterans. ${ }^{77}$ The study reported that the use of VR-EBT is feasible and acceptable to clinicians; its main conclusions suggest that they perceive VR-EBT as a step forward in treatment. It would be very acceptable to veterans. These results are promising, but it would be desirable to explore this important issue in further studies.

The big challenge is to bring these findings to the $3.6 \%$ of the world's population that, according to the World Health Organization, has suffered from PTSD. A recent cross-national survey in 21 countries $^{78}$ pointed out that more than $10 \%$ of participants reported witnessing violence $(21.8 \%)$ or experiencing interpersonal violence (18.8\%), accidents (17.7\%), exposure to war $(16.2 \%)$, or trauma to a loved one $(12.5 \%)$. These data reveal that it is important to conduct studies related to the use of VR-EBT for the treatment of PTSD. In this sense, various research groups studying the efficacy of VR-EBT in the treatment of PTSD are making great efforts to bring these treatments to mental health providers and patients in different parts of the world, for example, the United States (www.vrphobia.com, www.virtuallybetter.com), Spain (www.labpsitec.es, www.previsl.com), Belgium (www.vrphobia.eu), Mexico(www.solucionesvirtuales.com.mx), and Canada (w3.uqo.ca/cyberpsy, invirtuo.com).

Finally, it is necessary to interpret the results and conclusions of this review with caution because of some limitations. This review refers to a limited number of studies that met the inclusion criteria, which limits the type of studies reviewed to peer-reviewed studies. We made this decision in order to include studies with the highest quality possible; however, we recognize that, following these criteria, studies with nonsignificant results could be underreported. Future reviews should consider these aspects. 


\section{Disclosure}

The authors report no conflicts of interest in this work.

\section{References}

1. American Psychiatric Asociation. Diagnostic and statistical manual of mental disorders (DSM-5). 5 ed. Arlington, VA: American Psychiatric Asociation; 2013.

2. Chambless DL, Baker MJ, Baucom DH, Beutler LE, Calhoum KS, Crits-Christoph P. Update on empirically validated therapies, II. The Clinical Psychologist. 1998;51:3-16.

3. Rapaport MH, Clary C, Fayyad R, Endicott J. Quality-of-life impairment in depressive and anxiety disorders. American Journal of Psychiatry. 2005;162(6):1171-1178.

4. National Institute for Clinical Excellence. Post-traumatic stress disorder (PTSD): The management of PTSD in adults and children in primary and secondary care (clinical guideline 26). London, UK: National Institute for Clinical Excellence; 2005.

5. Foa EB, Gillihan SJ, Bryant RA. Challenges and successes in dissemination of evidence-based treatments for posttraumatic stress: Lessons learned from prolonged exposure therapy for PTSD. Psychological Science in the Public Interest, Supplement. 2013;14(2): 65-111.

6. Foa EB, Kozak MJ. Emotional Processing of Fear. Exposure to Corrective Information. Psychological Bulletin. 1986;99(1):20-35.

7. Foa EB, Hembree EA, Rothbaum BO. Prolonged exposure therapy for PTSD: Emotional processing of traumatic experiences: Therapist guide. New York: Oxford University Press; 2007.

8. McLean CP, Foa EB. The use of prolonged exposure therapy to help patients with post-traumatic stress disorder. Clinical Practice. 2014;11(2):233-241.

9. McLean CP, Foa EB. Dissemination and implementation of prolonged exposure therapy for posttraumatic stress disorder. Journal of Anxiety Disorders. 2013;27(8):788-792.

10. Eftekhari A, Ruzek JI, Crowley JJ, Rosen CS, Greenbaum MA, Karlin BE. Effectiveness of national implementation of prolonged exposure therapy in veterans affairs care. JAMA Psychiatry. 2013;70(9): 949-955.

11. Nayak N, Powers MB, Foa EB. Empirically Supported Psychological Treatments: Prolonged Exposure. The Oxford Handbook of Traumatic Stress Disorders. 2012.

12. Foa EB. Prolonged exposure therapy: Past, present, and future. Depression and Anxiety. 2011;28(12):1043-1047.

13. Powers MB, Halpern JM, Ferenschak MP, Gillihan SJ, Foa EB. A metaanalytic review of prolonged exposure for posttraumatic stress disorder. Clinical Psychology Review. 2010;30(6):635-641.

14. Karlin BE, Ruzek JI, Chard KM, et al. Dissemination of evidencebased psychological treatments for posttraumatic stress disorder in the Veterans Health Administration. Journal of Traumatic Stress. 2010;23(6):663-673.

15. Rauch SAM, Grunfeld TEE, Yadin E, Cahill SP, Hembree E, Foa EB. Changes in reported physical health symptoms and social function with prolonged exposure therapy for chronic posttraumatic stress disorder. Depression and Anxiety. 2009;26(8):732-738.

16. Foa EB, Rothbaum BO, Riggs DS, Murdock TB. Treatment of Posttraumatic Stress Disorder in Rape Victims: A Comparison Between Cognitive-Behavioral Procedures and Counseling. Journal of Consulting and Clinical Psychology. 1991;59(5):715-723.

17. Foa EB, Dancu CV, Hembree EA, Jaycox LH, Meadows EA, Street GP. A comparison of exposure therapy, stress inoculation training, and their combination for reducing posttraumatic stress disorder in female assault victims. Journal of Consulting and Clinical Psychology. 1999;67(2):194-200.
8. Foa EB, Hembree EA, Cahill SP, et al. Randomized trial of prolonged exposure for posttraumatic stress disorder with and without cognitive restructuring: Outcome at academic and community clinics. Journal of Consulting and Clinical Psychology. 2005;73(5):953-964.

19. Ruzek JI, Eftekhari A, Rosen CS, et al. Factors related to clinician attitudes toward prolonged exposure therapy for PTSD. Journal of Traumatic Stress. 2014;27(4):423-429.

20. Becker CB, Zayfert C, Anderson E. A survey of psychologists' attitudes towards and utilization of exposure therapy for PTSD. Behaviour Research and Therapy. 2004;42(3):277-292.

21. Hembree EA, Foa EB, Dorfan NM, Street GP, Kowalski J, Tu X. Do Patients Drop Out Prematurely from Exposure Therapy for PTSD? Journal of Traumatic Stress. 2003;16(6):555-562.

22. American Psychological Association. Evidence-based practice in psychology. American Psychologist. 2006;61(4):271-285.

23. American Psychological Association. Research-Supported Psychological Treatments. 2013; http://www.div12.org/psychological-treatments/, 2015.

24. McLean CP, Foa EB. Prolonged exposure therapy for post-traumatic stress disorder: A review of evidence and dissemination. Expert Review of Neurotherapeutics. 2011;11(8):1151-1163.

25. Nacasch N, Foa EB, Huppert JD, et al. Prolonged exposure therapy for combat- and terror-related posttraumatic stress disorder: a randomized control comparison with treatment as usual. The Journal of Clinical Psychiatry. 2011;72(9):1174-1180.

26. Bryant RA, Moulds ML, Guthrie RM, et al. A randomized controlled trial of exposure therapy and cognitive restructuring for posttraumatic stress disorder. Journal of Consulting and Clinical Psychology. 2008;76(4):695-703.

27. Taylor S, Thordarson DS, Maxfield L, Fedoroff IC, Lovell K, Ogrodniczuk J. Comparative efficacy, speed, and adverse effects of three PTSD treatments: Exposure therapy, EMDR, and relaxation training. Journal of Consulting and Clinical Psychology. 2003;71(2):330-338.

28. Resick PA, Nishith P, Weaver TL, Astin MC, Feuer CA. A comparison of cognitive-processing therapy with prolonged exposure and a waiting condition for the treatment of chronic posttraumatic stress disorder in female rape victims. Journal of Consulting and Clinical Psychology. 2002;70(4):867-879.

29. Botella C, Quero S, Baños RM, Perpiñá C, García-Palacios A. Virtual reality and psychotherapy. In: Riva G, Botella C, Légeron P, Optale G, eds. Cybertherapy. Vol 99. Amsterdam: IOS Press; 2004:37-52.

30. Powers MB, Emmelkamp PMG. Virtual reality exposure therapy for anxiety disorders: A meta-analysis. Journal of Anxiety Disorders. 2008; 22(3):561-569.

31. Opriş D, Pintea S, García-Palacios A, Botella C, Szamosközi Ş, David D. Virtual reality exposure therapy in anxiety disorders: A quantitative meta-analysis. Depression and Anxiety. 2012;29(2):85-93.

32. Parsons TD, Rizzo AA. Affective outcomes of virtual reality exposure therapy for anxiety and specific phobias: A meta-analysis. Journal of Behavior Therapy and Experimental Psychiatry. 2008; 39(3):250-261.

33. Turner WA, Casey LM. Outcomes associated with virtual reality in psychological interventions: where are we now? Clinical Psychology Review. 2014;34(8):634-644.

34. Gregg L, Tarrier N. Virtual reality in mental health. A review of the literature. Social Psychiatry and Psychiatric Epidemiology. 2007;42(5):343-354.

35. Serrano B, Botella C, Wiederhold B, Baños RM. Virtual reality and anxiety disorders treatment: Evolution and future perspectives. In: Bouchard S, Sharkey P, eds. Virtual reality technologies for health and clinical applications: Psychological and neurocognitive interventions: Springer; in press.

36. Krijn M, Emmelkamp PMG, Olafsson RP, Biemond R. Virtual reality exposure therapy of anxiety disorders: A review. Clinical Psychology Review. 2004;24(3):259-281.

37. Meyerbröker K, Emmelkamp PMG. Virtual reality exposure therapy in anxiety disorders: A systematic review of process-and-outcome studies. Depression and Anxiety. 2010;27(10):933-944. 
38. García-Palacios A, Botella C, Hoffman H, Fabregat S. Comparing acceptance and refusal rates of virtual reality exposure vs. in vivo exposure by patients with specific phobias. CyberPsychology and Behavior. 2007;10(5):722-724.

39. Segal R, Bhatia M, Drapeau M. Therapists' perception of benefits and costs of using virtual reality treatments. Cyberpsychology, Behavior, and Social Networking. 2011;14(1-2):29-34.

40. Motraghi TE, Seim RW, Meyer EC, Morissette SB. Virtual reality exposure therapy for the treatment of posttraumatic stress disorder: A methodological review using CONSOR. International Journal of Clinical Psychology. 2014;70:197-208.

41. Gonçalves R, Pedrozo AL, Coutinho ESF, Figueira I, Ventura P. Efficacy of Virtual Reality Exposure Therapy in the Treatment of PTSD: A Systematic Review. PLoS One. 2012;7(12).

42. García-Palacios A, Botella C, Baños R, Guillén V, Navarro MV. Inclusion of virtual reality: A rationale for the use of VR in the treatment of PTSD In: Safir MP, Wallach HS, Rizzo A, eds. Future directions in post-traumatic stress disorder. Prevention, diagnosis, and treatment. Los Angeles: Springer; 2014:275-288.

43. Wiederhold BK, Wiederhold M. Virtual reality for posttraumatic stress disorder. In: Wiederhold BK, Bouchard S, eds. Advances in virtual reality and anxiety disorders. Series in anxiety and related disorders New York: Springer; 2014:211-234.

44. Becker CB, Darius E, Schaumberg K. An analog study of patient preferences for exposure versus alternative treatments for posttraumatic stress disorder. Behaviour Research and Therapy. 2007;45(12): 2861-2873.

45. Rothbaum BO, Hodges L, Alarcon R, et al. Virtual reality exposure therapy for PSTD Vietnam veterans: A case study. Journal of Traumatic Stress. 1999;12(2):263-271.

46. Hodges LF, Rothbaum BO, Alarcon R, et al. A virtual environment for the treatment of chronic combat-related post-traumatic stress disorder. Cyberpsychology and Behavior. 1999;2(1):7-14.

47. Rizzo A, Pair J, McNerney PJ, et al. Development of a VR therapy application for Iraq war military personnel with PTSD. Studies in Health Technology and Informatics. Vol 1112005:407-413.

48. Difede J, Cukor J, Jayasinghe N, et al. Virtual reality exposure therapy for the treatment of posttraumatic stress disorder following September 11, 2001. Journal of Clinical Psychiatry. 2007;68(11):1639-1647.

49. Josman N, Somer E, Reisberg A, Weiss PL, Garcia-Palacios A, Hoffman H. BusWorld: Designing a virtual environment for post-traumatic stress disorder in Israel: A protocol. Cyberpsychology and Behavior. 2006;9(2):241-244.

50. Gamito P, Oliveira J, Rosa P, et al. PTSD elderly war veterans: A clinical controlled pilot study. Cyberpsychology, Behavior, and Social Networking. 2010;13(1):43-48.

51. Wood DP, Murphy J, Center K, et al. Combat-related post-traumatic stress disorder: A case report using virtual reality exposure therapy with physiological monitoring. Cyberpsychology and Behavior. 2007;10(2):309-315.

52. Cárdenas-López G, de la Rosa A. Post-traumatic stress disorder treatment with virtual reality exposure for criminal violence: A case study in assault with violence. International Journal on Disability and Human Development. 2011;10(4):379-383.

53. Baños RM, Botella $\mathrm{C}$, Guillen V, et al. An adaptive display to treat stress-related disorders: EMMA's world. British Journal of Guidance and Counselling. 2009;37(3):347-356.

54. Rothbaum BO, Price M, Jovanovic T, et al. A randomized, doubleblind evaluation of D-cycloserine or alprazolam combined with virtual reality exposure therapy for posttraumatic stress disorder in Iraq and Afghanistan war veterans. American Journal of Psychiatry. 2014;171(6):640-648.

55. Botella C, García-Palacios A, Guillen V, Baños RM, Quero S, Alcaniz M. An adaptive display for the treatment of diverse trauma PTSD victims. Cyberpsychology, Behavior, and Social Networking. 2010;13(1):67-71.
56. Mulligan A, Hall L, Raphael E. Peer review in a changing world: An international study measuring the attitudes of researchers. Journal of the American Society for Information Science and Technology. 2013;64(1):132-161.

57. Barlow DH, Hoffman SG. Efficacy and dissemination of psychological treatments. In: Clark DM, Fairburn CG, eds. Science and practice of cognitive behavior therapy. Oxford, R.U: Oxford University Press; 1997:95-117.

58. Difede J, Cukor J, Wyka K, et al. D-cycloserine augmentation of exposure therapy for post-traumatic stress disorder: A pilot randomized clinical trial. Neuropsychopharmacology. 2014;39(5):1052-1058.

59. Roy MJ, Costanzo ME, Blair JR, Rizzo AA. Compelling evidence that exposure therapy for PTSD normalizes brain function. Studies in Health Technology and Informatics. 2014:2014;199:61-65.

60. De la Rosa A, Cárdernas-López G. Posttraumatic stress disorder: Efficacy of a treatment program using virtual reality for victims of criminal violence in Mexican population. Anuario de Psicologia. 2012;42(3):377-391.

61. Ready DJ, Gerardi RJ, Backscheider AG, Mascaro N, Rothbaum BO. Comparing virtual reality exposure therapy to present-centered therapy with 11 U.S. Vietnam veterans with PTSD. Cyberpsychology, Behavior, and Social Networking. 2010;13(1):49-54.

62. Blake DD, Weathers FW, Nagy LM, et al. The development of a Clinician Administered PTSD Scale. Journal of Trauma Stress. 1995;8: 75-90.

63. McLay RN, McBrien C, Wiederhold MD, Wiederhold BK. Exposure therapy with and without virtual reality to treat PTSD while in the combat theater: A parallel case series. Cyberpsychology, Behavior, and Social Networking. 2010;13(1):37-42.

64. McLay RN, Wood DP, Webb-Murphy JA, et al. A randomized, controlled trial of virtual reality-graded exposure therapy for post-traumatic stress disorder in active duty service members with combat-related post-traumatic stress disorder. Cyberpsychology, Behavior, and Social Networking. 2011;14(4):223-229.

65. Miyahira SD, Folen RA, Hoffman HG, Garcia-Palacios A, Spira JL, Kawasaki M. The effectiveness of VR exposure therapy for PTSD in returning warfighters. Studies in Health Technology and Informatics. 2012;181:128-132.

66. Rey B, Montesa J, Alcañiz M, Baños R, Botella C. A preliminary study on the use of an adaptive display for the treatment of emotional disorders. PsychNology Journal. 2005;3(1):101-112.

67. Baños RM, Guillen V, Quero S, García-Palacios A, Alcaniz M, Botella C. A virtual reality system for the treatment of stress-related disorders: A preliminary analysis of efficacy compared to a standard cognitive behavioral program. International Journal of Human Computer Studies. 2011;69(9):602-613.

68. Beck JG, Palyo SA, Winer EH, Schwagler BE, Ang EJ. Virtual reality exposure therapy for PTSD symptoms after a road accident: an uncontrolled case series. Behavior Therapy. 2007;38(1):39-48.

69. Rizzo A, Difede J, Rothbaum BO, et al. Development and early evaluation of the virtual Iraq/Afghanistan exposure therapy system for combat-related PTSD. Psychiatric and Neurologic Aspects of War. 2010;1208:114-125.

70. Cárdenas-López G, de la Rosa A, Durán-Baca X, Bouchard S. Virtual reality PTSD treatment program for civil victims of criminal violence. In: Cipresso P, Serino S, eds. Virtual reality: technologies, medical applications and challenges. Hauppauge (NY): Nova Science Publishers; 2015:269-290.

71. Gerardi M, Rothbaum BO, Ressler K, Heekin M, Rizzo A. Virtual reality exposure therapy using a virtual Iraq: Case report. Journal of Traumatic Stress. 2008;21(2):209-213.

72. Reger GM, Gahm GA. Virtual reality exposure therapy for active duty soldiers. Journal of Clinical Psychology. 2008;64(8):940-946.

73. McLay R, Ram V, Murphy J, et al. Effect of virtual reality PTSD treatment on mood and neurocognitive outcomes. Cyberpsychology, Behavior, and Social Networking. 2014;17(7):439-446. 
74. Tarnanas I, Manos G. A clinical protocol for the development of a virtual reality behavioral training in disaster exposure and relief. In: Wiederhold BK, Bouchard S, Riva G, eds. Annual Review of CyberTherapy and Telemedicine. Vol 2. San Diego: Interactive Media Intitute; 2004:78-84.

75. López-Soler C, Castro M, Alcántara M, Botella C. The virtual reality system EMMA-Childhood in the psychological treatment of a minor with posttraumatic stress disorder. Revista de Psicopatologia y Psicología Clínica. 2011;16(3):189-206.
76. Bouchard S, Guitard T, Bernier F, Robillard G. Virtual reality and the training of military personnel to cope with acute stressors. Studies in Computational Intelligence. Vol 3372011:109-128.

77. Kramer TL, Pyne JM, Kimbrell TA, Savary PE, Smith JL, Jegley SM. Clinician perceptions of virtual reality to assess and treat returning veterans. Psychiatric Services. 2010;61(11):1153-1156.

78. Stein DJ, Chiu WT, Hwang I, et al. Cross-national analysis of the associations between traumatic events and suicidal behavior: findings from the WHO World Mental Health Surveys. PloS one. 2010;13(5):e10574.

\section{Publish your work in this journal}

Neuropsychiatric Disease and Treatment is an international, peerreviewed journal of clinical therapeutics and pharmacology focusing on concise rapid reporting of clinical or pre-clinical studies on a range of neuropsychiatric and neurological disorders. This journal is indexed on PubMed Central, the 'PsycINFO' database and CAS, and is the official journal of The International Neuropsychiatric Association (INA). The manuscript management system is completely online and includes a very quick and fair peer-review system, which is all easy to use. Visit http://www.dovepress.com/testimonials.php to read real quotes from published authors.

Submit your manuscript here: http://www.dovepress.com/neuropsychiatric-disease-and-treatment-journal 